\title{
Brauer relations in finite groups II: Quasi-elementary groups of order $p^{a} q$
}

\author{
Alex Bartel and Tim Dokchitser \\ Communicated by Robert M. Guralnick
}

\begin{abstract}
This is the second in a series of papers investigating the space of Brauer relations of a finite group, the kernel of the natural map from its Burnside ring to the rational representation ring. The first paper classified all primitive Brauer relations, that is those that do not come from proper subquotients. In the case of quasi-elementary groups the description is intricate, and it does not specify groups that have primitive relations in terms of generators and relations. In this paper we provide such a classification in terms of generators and relations for quasi-elementary groups of order $p^{a} q$.
\end{abstract}

\section{Introduction}

Let $G$ be a finite group. Recall that there is a natural map from the Burnside ring $B(G)$ of $G$ to the rational representation ring $R_{\mathbb{Q}}(G)$, which sends a $G$-set to the corresponding permutation representation. In [1], we have described the kernel $K(G)$ of this map by giving a set of generators, following the work of Bouc [2] who had described this kernel for $p$-groups. The purpose of this paper is to make our description more explicit in the most difficult case, that of quasi-elementary groups.

We will write elements of $B(G)$ as $\mathbb{Z}$-linear combinations $\sum_{H \leq G} a_{H} H$, using the identification between transitive $G$-sets and conjugacy classes of subgroups of $G$ that maps a $G$-set to a point stabiliser. We call an element of $K(G)$ a Brauer relation.

If $H \leq G$, then there is an induction map $B(H) \rightarrow B(G)$, which yields an induction map $K(H) \rightarrow K(G)$. We say that a Brauer relation of $G$ is induced from $H$ if it is in the image of this map. Also, if $N \triangleleft G$, then there is an inflation map $B(G / N) \rightarrow B(G)$, which induces a map $K(G / N) \rightarrow K(G)$. We say that

The first author was partially supported by the EPSRC and is partially supported by a from the Royal Commission for the Exhibition of 1851, and the second author is supported by a Royal Society University Research Fellowship. Parts of this research were done at St Johns College, Robinson College and DPMMS in Cambridge, CRM in Barcelona, and Postech University in Pohang. We would like to thank these institutions for their hospitality and financial support. 
a Brauer relation of $G$ is lifted from $G / N$ if it is in the image of this inflation map. We call a relation imprimitive if it is a linear combination of relations that are induced and/or lifted from proper subquotients of $G$ and primitive otherwise. The quotient of $K(G)$ by the subgroup consisting of imprimitive relations will be denoted by $\operatorname{Prim}(G)$.

The main result of [1] is a group theoretic criterion for $\operatorname{Prim}(G)$ to be non-trivial and a determination of the group structure of $\operatorname{Prim}(G)$ and of a set of generators. This criterion is most complicated when $G$ is quasi-elementary, i.e. when it is of the form $G=C \rtimes P$, where $C$ is cyclic and $P$ is a $p$-group. Write $K$ for the kernel of the conjugation action of $P$ on $C$. It is shown in [1] that for such a group to have primitive relations, $C$ must be of square-free order, and $K$ must be either trivial, or isomorphic to $D_{8}$, or have normal $p$-rank one. When $K$ is trivial, a complete description of $\operatorname{Prim}(G)$ is provided by [1, Proposition 6.5]. Here, we explicitly describe the structure of those quasi-elementary groups for which $\operatorname{Prim}(G)$ is non-trivial in the case that the order of $C$ is prime and $K$ is non-trivial. We determine the general shape of a presentation for such groups in terms of generators and relations, thereby explicating the criterion of [1, Theorem 7.30] in this special case.

Theorem 1.1. Let $G=C \rtimes P$ be quasi-elementary, where $C$ is cyclic of prime order $q$ and $P$ is a p-group with $p \neq q$. Suppose that $K=\operatorname{ker}(P \rightarrow \operatorname{Aut} C)$ is either isomorphic to $D_{8}$ or has normal $p$-rank one. A necessary condition for $G$ to have primitive relations is that $P$ must be of the form $P=K \rtimes A$, where $A$ acts faithfully on $C$, and in particular is cyclic of order $p^{m}$, say, $A=\langle h\rangle$. Assume that this condition is satisfied. Let $K$ be generated by $c$ of order $p^{n}$ and possibly $x$ of order 2 or 4 as in Proposition 2.2, and define j, $k$ by $h c h^{-1}=c^{j}, h x h^{-1}=c^{k} x$. The following are all the cases in which $\operatorname{Prim}(G)$ is non-trivial, together with the group structure of $\operatorname{Prim}(G)$ :

(1) $K$ is cyclic, $p \neq 2, n \leq m ; \operatorname{Prim}(G) \cong C_{p}^{u}$, where $u=p-2$ if $n=1$ and $u=p-1$ otherwise.

(2) $K$ is cyclic, $p=2, j \neq-1$; moreover, either $1<n \leq m$, or $j \equiv 3(\bmod 4)$ and the order of $j$ in $\left(\mathbb{Z} / 2^{n} \mathbb{Z}\right)^{\times}$divides $2^{m-1}$; in either case, $\operatorname{Prim}(G) \cong C_{2}$.

(3) $K$ is generalised quaternion, $k$ is odd, and $n<m$; $\operatorname{Prim}(G) \cong C_{2}$.

(4) $K$ is dihedral, $k$ is even, $j \neq \pm 1$, and

$$
2^{n}\left|j^{2^{m-1}}-1, \quad 2^{n}\right| k\left(j^{2^{m-1}}-1\right) /(j-1) ;
$$

$\operatorname{Prim}(G) \cong C_{2}$ 
(5) $K$ is dihedral, $k$ is odd, and either $m>n$ or

$$
j \neq \pm 1, \quad 2^{n} \mid\left(j^{2^{m-1}}-1\right) /(j-1)
$$

$\operatorname{Prim}(G) \cong C_{2} \times C_{2}$ if $m>n$ and $j \neq \pm 1$, and $\operatorname{Prim}(G) \cong C_{2}$ otherwise.

Our strategy is equally suited to obtaining a concrete result of the same nature when $|C|$ has any other fixed number of prime divisors, although in the case under consideration some substantial simplifications occur, most notably Proposition 2.5.

\section{Some general results on the structure of $P$}

We recall the relevant results from [1]. Throughout, we assume that $G=C \rtimes P$, where $C$ is cyclic of prime order $q$ and $P$ is a $p$-group with $p \neq q$. The kernel of the conjugation action of $P$ on $C$ is denoted by $K$.

Proposition 2.1 ([1, Proposition 7.6]). If $G$ has a primitive relation, then $K$ is either trivial or isomorphic to $D_{8}$ or has normal p-rank one.

Recall that the normal $p$-rank of a group is defined as the maximal rank of a normal elementary abelian $p$-subgroup.

Proposition 2.2 ([3, Theorem 5.4.10]). Let $X$ be a p-group with normal p-rank one. Then $X$ is one of the following:

- the cyclic group $C_{p^{n}}=\left\langle c \mid c^{p^{n}}=1\right\rangle$,

- the dihedral group $D_{2^{n+1}}=\left\langle c, x \mid c^{2^{n}}=x^{2}=1, x c x=c^{-1}\right\rangle$ with $n \geq 3$,

- the generalised quaternion group, $Q_{2^{n+1}}=\left\langle c, x \mid c^{2^{n-1}}=x^{2}, x^{-1} c x=c^{-1}\right\rangle$ with $n \geq 2$,

- the semi-dihedral group $\mathrm{SD}_{2^{n+1}}=\left\langle c, x \mid c^{2^{n}}=x^{2}=1, x c x=c^{2^{n-1}-1}\right\rangle$ with $n \geq 3$.

Notation 2.3. If $K$ is trivial, then $\operatorname{Prim}(G)$ is described explicitly by [1, Proposition 6.5]. From now on, assume that $K$ is either isomorphic to $D_{8}$ or has normal $p$-rank one. In particular, $K$ contains a unique subgroup of order $p$ that is normal in $G$, which we will denote by $C_{p}^{z}$. Let $\mathscr{H}$ denote the set of those subgroups ${ }^{1}$ of $P$ that do not contain $C_{p}^{z}$, and let $\mathscr{H}_{m}$ be the set of elements of $\mathscr{H}$ of maximal size. Let $C_{K}$ be either $K$ if $K$ is cyclic, or a cyclic index two subgroup of $K$ that is

1 This definition slightly differs from the one in [1], where $\mathscr{H}$ was defined as a set of representatives of conjugacy classes of subgroups. 
normal in $G$ otherwise (such an index two subgroup is unique unless $K=Q_{8}$, in which case there may be three such groups, see [1, Notation 7.9] for details). Set $\bar{C}_{K}=C C_{K}$.

Lemma 2.4 ([1, Lemma 7.27]). The group $\operatorname{Prim}(G)$ is generated by relations of the form

$$
\Theta=\sum_{\tilde{C} \leq \bar{C}_{K}} \mu(|\tilde{C}|)\left(\tilde{C} H-\tilde{C} H^{\prime}\right),
$$

for $H, H^{\prime} \in \mathscr{H}_{m}$, where $\mu$ denotes the Möbius function.

Proposition 2.5. If $G$ has a primitive relation, then $P$ is a semi-direct product by $K, P=K \rtimes A$, where $A$ acts faithfully on $C$.

Proof. Since $|C|$ is prime, Aut $C$ is cyclic. So for a subgroup $H$ of $P$, either the image of $H$ under $P \rightarrow$ Aut $C$ is equal to that of $P$, or $H$ is contained in the preimage under $P \rightarrow$ Aut $C$ of the unique index $p$ subgroup of Aut $C$. Thus, either $\mathscr{H}_{m}$ contains a subgroup whose image under $P \rightarrow$ Aut $C$ is equal to that of $P$, or the relations of Lemma 2.4 are all imprimitive by [1, Proposition 3.7].

Suppose for the rest of the proof that $\mathscr{H}$ contains a subgroup $A$ of $P$ whose image in Aut $C$ is equal to that of $P$. If $A$ intersects $K$ trivially, then $P$ must be a semi-direct product $K \rtimes A$. In particular, this proves the claim in the cases that $K$ is cyclic or generalised quaternion, since in those cases intersecting $K$ trivially is equivalent to not containing $C_{p}^{z}$.

Suppose that $K$ is dihedral or semi-dihedral (in particular, $P$ is a 2-group). Then $P$ is a semi-direct product by $C_{K}$. But since the automorphism of $C_{K}$ given by conjugation by a non-central involution of $K$ is not divisible by 2 in Aut $C_{K}$, this implies that $P$ is a semi-direct product by $K$ and the proof of the proposition is complete.

Lemma 2.6 ([1, Corollary 7.4]). If $K$ is non-trivial and $P$ has cyclic centre, then $G$ has no primitive relations.

Hypothesis 2.7. From now on, assume that $P$ is a semi-direct product by $K$ and that the conjugation action of the quotient on $K$ has a non-trivial kernel.

Note that if $|K|=p$, and in particular $P$ is a direct product, then [1, Proposition 7.29] implies that $\operatorname{Prim}(G) \cong \mathbb{F}_{p}^{p-2}$. In the case that $|K| \neq p$, we recall the description of $\operatorname{Prim}(G)$ in the special case under consideration that we want to make explicit. 
Theorem 2.8 ([1, Theorem 7.30]). Assume that $|K|>p$. Define a graph $\Gamma$ whose vertices are the elements of $\mathscr{H}_{m}$ and with an edge between $H, H^{\prime} \in \mathscr{H}_{m}$ if one of the following applies:

(1) the subgroup generated by $H$ and $H^{\prime}$ is a proper subgroup of $P$,

(2) the intersection $H \cap H^{\prime}$ is of index $p$ in $H$ and in $H^{\prime}$, and $H H^{\prime} / H \cap H^{\prime}$ is either dihedral, or the Heisenberg group of order $p^{3}$.

Let $d$ be the number of connected components of $\Gamma$. Then $\operatorname{Prim}(G) \cong\left(C_{p}\right)^{d-1}$, generated by relations $\Theta=\sum_{\tilde{C} \leq \bar{C}_{K}} \mu(|\tilde{C}|)\left(\tilde{C} H-\tilde{C} H^{\prime}\right)$ for $H, H^{\prime} \in \mathscr{H}_{m}$ corresponding to distinct connected components of the graph.

Remark 2.9. If $H$ is a proper subgroup of $P$, then its image in the Frattini quotient of $P$ is also a proper subgroup. Since the Frattini quotient is abelian, $P$ acts trivially on it by conjugation, so if $H^{\prime}$ is any conjugate of $H, H$ and $H^{\prime}$ never generate the whole of $P$. Thus, conjugate subgroups always lie in the same connected component of $\Gamma$.

Notation 2.10. We will retain the notation $\Gamma$ for the graph described in Theorem 2.8 for the rest of the paper.

\section{$3 \quad K$ is cyclic}

Let

$$
K=\left\langle c \mid c^{p^{n}}=1\right\rangle, \quad n \geq 2 .
$$

The only subgroup of $K$ that does not contain $C_{p}^{z}$ is the trivial group, so $\mathscr{H}$ consists of subgroups of $P$ that intersect $K$ trivially. We have $P=K \rtimes A$ where $A=\langle h\rangle$ is cyclic of order $p^{m}$, acts faithfully on $C$ and acts as $h c h^{-1}=c^{j}$ on $K$ with $j$ of order dividing $p^{m-1}$ in $\left(\mathbb{Z} / p^{n} \mathbb{Z}\right)^{\times}$and in particular $j \equiv 1(\bmod p)$.

All elements of $\mathscr{H}_{m}$ are of the form $H_{\alpha}=\left\langle c^{\alpha} h\right\rangle$ for $0 \leq \alpha \leq p^{n}-1$. Conversely, such an $H_{\alpha}$ is a complement of $C \times K$ in $G$ if and only if $H_{\alpha} \cap K=\{1\}$ if and only if $c^{\alpha} h$ has order $p^{m}$.

Lemma 3.1. Let $l$ be a prime and let $n \equiv 1(\bmod l)$ if $l$ is odd and $n \equiv 1(\bmod 4)$ if $l=2$. Then

$$
v_{l}\left(n^{s}-1\right)=v_{l}(n-1)+v_{l}(s)
$$

for any positive integer $s$, where $v_{l}$ is the normalised $l$-adic valuation.

Proof. Set $r=l$ if $l$ is odd and $r=4$ if $l=2$. The isomorphism

$$
\exp :\left(r \mathbb{Z}_{l},+\right) \rightarrow\left(1+r \mathbb{Z}_{l}, \times\right)
$$


preserves the standard filtrations on both hand sides, i.e. it satisfies

$$
v_{l}(t)=v_{l}(\exp (t)-1) .
$$

Raising to the $s$-th power on the right hand side corresponds to multiplying by $s$ on the left hand side and the result follows.

Now,

$$
\left(c^{\alpha} h\right)^{p^{m}}=c^{\alpha\left(j^{p^{m}-1}+j^{p^{m}-2}+\cdots+j+1\right)} h^{p^{m}}= \begin{cases}c^{\alpha \frac{j^{p^{m}}-1}{j-1}}, & j \neq 1, \\ c^{\alpha p^{m}}, & j=1 .\end{cases}
$$

It follows that if $\alpha \equiv \beta(\bmod p)$, then $H_{\alpha} \in \mathscr{H}_{m}$ if and only if $H_{\beta} \in \mathscr{H}_{m}$. Moreover, two such groups generate a proper subgroup of $P$, so the corresponding vertices of $\Gamma$ lie in one connected component of the graph. Thus, each connected component is represented by some $H_{\alpha}$ with $0 \leq \alpha \leq p-1$. If $p$ is odd, then Lemma 3.1 implies that for any $\alpha \in\{1, \ldots, p-1\}, H_{\alpha}$ is a complement if and only if $m \geq n$. If on the other hand $p=2$, then $H_{1}$ is a complement if and only if either $j \equiv 3(\bmod 4)$ (since the order of $j \in\left(\mathbb{Z} / 2^{n} \mathbb{Z}\right)^{\times}$divides $\left.2^{m-1}\right)$ or $j \equiv 1(\bmod 4)$ and $m \geq n$ (by Lemma 3.1). In particular, if $p$ is odd and $n>m$ or if $p=2, j \equiv 1(\bmod 4)$ and $n>m$, then the graph $\Gamma$ has only one connected component, so $G$ has no primitive relations.

From now on assume that either $n \leq m$ or $p=2, j \equiv 3(\bmod 4)$, and the order of $j$ in $\left(\mathbb{Z} / 2^{n} \mathbb{Z}\right)^{\times}$divides $2^{m-1}$.

If $\alpha \neq \equiv \beta(\bmod p)$, then $H_{\alpha}$ and $H_{\beta}$ together generate $P$. Since $n>1$, the group $P / H_{\alpha} \cap H_{\beta}$ cannot be isomorphic to the Heisenberg group of order $p^{3}$, having exponent strictly bigger than $p$. Finally, $P / H_{\alpha} \cap H_{\beta}$ can only be isomorphic to a dihedral group if $p=2$ and $j=-1$, in which case any such quotient is dihedral. This completes the proof of cases (1) and (2) of Theorem 1.1.

In all the remaining cases, we have $p=2$.

\section{$4 K$ is semi-dihedral}

A presentation of $P$ by generators and relations is given by

$$
\begin{gathered}
K \rtimes A=\langle x, c, h| x^{2}=c^{2^{n}}=h^{2^{m}}=1, x c x=c^{2^{n-1}-1}, \\
h x h^{-1}=c^{k} x, h c h^{-1}=c^{j}, \\
\left.n \geq 3, j \in\left(\mathbb{Z} / 2^{n} \mathbb{Z}\right)^{\times}, k \in \mathbb{Z} / 2^{n} \mathbb{Z}\right\rangle .
\end{gathered}
$$

Here, $k$ must be even, since if $z$ is the central involution of $K$, then

$$
h=x(x h x) x=x z^{k} c^{-k} h x=z^{k} x c^{-k} c^{k} x h=z^{k} h .
$$


The only conjugacy class of non-trivial subgroups of $K$ that do not contain the subgroup $C_{p}^{z}=\langle z\rangle$ is that of non-central involutions, represented by $\langle x\rangle$, so the elements of $\mathscr{H}$ are subgroups of $P$ that either intersect $K$ trivially or those that intersect it in $\left\langle c^{r} x\right\rangle$ for some $r \in \mathbb{Z} / 2^{n} \mathbb{Z}$.

Since $k$ is even, $h$ fixes a non-central involution, $h c^{i} x h^{-1}=c^{k i+k} x$, and $i$ can be chosen such that $c^{k i+k}=1$. So without loss of generality, replacing $x$ by $c^{i} x$ for such an $i$, assume that $k=0$. In particular, elements of $\mathscr{H}_{m}$ have size $2^{m+1}$ and are isomorphic to $C_{2} \times C_{2^{m}}$.

We claim that all elements of $\mathscr{H}_{m}$ are contained in $\left\langle c^{2}, h, x\right\rangle$, so that any two of them generate a proper subgroup of $P$, which will show that $G$ has no primitive relations when $K$ is semi-dihedral. Indeed, any element of $\mathscr{H}_{m}$ is of the form $\left\langle c^{r} h, c^{s} x\right\rangle$, with the two generators commuting, and it suffices to show that both $r$ and $s$ must be even. Since $1=\left(c^{s} x\right)^{2}=z^{s}, s$ must be even. Moreover, the condition that the two generators commute implies that

$$
\begin{gathered}
c^{r} h c^{s} x\left(c^{r} h\right)^{-1}\left(c^{s} x\right)^{-1}=z^{-r} c^{2 r+(j-1) s}=1 \\
\Longrightarrow 2 r+(j-1) s \equiv 0 \quad(\bmod 4) \\
\Longrightarrow r \text { is even }
\end{gathered}
$$

as required.

\section{$5 \quad K$ is generalised quaternion}

A presentation for $P$ by generators and relations is

$$
\begin{gathered}
P=\langle x, c, h| c^{2^{n}}=h^{2^{m}}=1, x^{2}=c^{2^{n-1}}, x c x^{-1}=c^{-1}, \\
h x h^{-1}=c^{k} x, h c h^{-1}=c^{j}, \\
\left.n \geq 2, j \in\left(\mathbb{Z} / 2^{n} \mathbb{Z}\right)^{\times}, k \in \mathbb{Z} / 2^{n} \mathbb{Z}\right\rangle .
\end{gathered}
$$

Since every non-trivial subgroup of $K$ contains $C_{2}^{z}, \mathscr{H}$ consists of subgroups of $P$ that intersect $K$ trivially. Note that it follows from the fact that $j$ is odd that the parity of $k$ is independent of the choice of $x$.

Case 1: $k$ even. Then $h, x$, and $c$ are independent in the Frattini quotient of $P$, which is therefore three-dimensional. Since elements of $\mathscr{H}$ are cyclic, no two of them can generate $P$, so the graph $\Gamma$ has only one connected component and $G$ has no primitive relations.

Case 2: $\boldsymbol{k}$ odd. The Frattini quotient of $P$ is then two-dimensional and has exactly three lines, generated by the images of $x, h$, and $x h$, respectively. Only two of these can be images of elements of $\mathscr{H}_{m}$, the lines generated by the images of $h$ and $x h$. The structure of $K$ implies that two elements of $\mathscr{H}_{m}$ never satisfy 
condition (2) of Theorem 2.8, so we deduce that there is no edge between the vertices of $\Gamma$ corresponding to $H, H^{\prime} \in \mathscr{H}_{m}$ if and only if their images in the Frattini quotient of $P$ are the lines generated by $h$ and $x h$, respectively, and that $\operatorname{Prim}(G) \cong C_{2}$ if and only if there exists an $H \in \mathscr{H}_{m}$ with image $\langle x h\rangle$ in the Frattini quotient, and is trivial otherwise.

An arbitrary element of $\mathscr{H}_{m}$ is of the form $\left\langle h x^{\delta} c^{r}\right\rangle$ for $\delta \in\{0,1\}$ and $0 \leq r \leq$ $2^{m}-1$, and a group of this form is in $\mathscr{H}$ if and only if $h x^{\delta} c^{r}$ has order $2^{m}$ (in general, the order is greater than or equal to $2^{m}$ ). Such a group has image equal to that of $\langle x h\rangle$ in the Frattini quotient if and only if $\delta=1$. We have

$$
\left(h x c^{r}\right)^{2^{m}}=c^{\left(j^{2^{m}-1}+j^{2^{m}-3}+\cdots+j\right)(i-r+j r)}= \begin{cases}c^{\text {odd } \cdot 2^{m-1}}, & j^{2}=1, \\ c^{\text {odd } \cdot\left(j^{2}-1\right) /\left(j^{2}-1\right)}, & j^{2} \neq 1 .\end{cases}
$$

By Lemma 3.1, the 2-adic valuation of the exponent is $m-1$ in both cases, so the right hand side is 1 if and only if $m-1 \geq n$. That condition also insures that $A$ acts non-faithfully on $K$. This completes the proof of case (3) of Theorem 1.1.

\section{$6 \quad K$ is dihedral}

In this case $P$ is given by generators and relations as

$$
\begin{gathered}
P=\langle x, c, h| c^{2^{n}}=h^{2^{m}}=x^{2}=1, x c x=c^{-1}, \\
h x h^{-1}=c^{k} x, h c h^{-1}=c^{j}, \\
\left.n \geq 2, j \in\left(\mathbb{Z} / 2^{n} \mathbb{Z}\right)^{\times}, k \in \mathbb{Z} / 2^{n} \mathbb{Z}\right\rangle .
\end{gathered}
$$

We have the following identities, which we will use repeatedly:

$$
\begin{aligned}
& h^{i} x h^{-i}=c^{k\left(j^{i-1}+j^{i-2}+\cdots+j+1\right)} x= \begin{cases}c^{k \frac{j^{i}-1}{j-1}} x, & j \neq 1, \\
c^{k i} x, & j=1,\end{cases} \\
& h^{i} c h^{-i}=c^{j^{i}} .
\end{aligned}
$$

Recall that by Hypothesis $2.7, h^{2^{m-1}}$ acts trivially on $K$. Equations (6.1) and (6.2) imply that this condition is equivalent to

$2^{n} \mid j^{2^{m-1}}-1$, i.e. $2^{n} \mid\left(j^{2}-1\right) 2^{m-2}$, and $\begin{cases}2^{n} \mid k 2^{m-1}, & j=1, \\ 2^{n} \mid k\left(j^{2^{m-1}}-1\right) /(j-1), & j \neq 1,\end{cases}$

which we assume throughout. For any $i \in \mathbb{Z} / 2^{n} \mathbb{Z}$, we have

$$
\begin{aligned}
& \left(c^{i} h\right)^{2^{m}}=c^{i\left(j^{2^{m}-1}+j^{2^{m}-2}+\cdots+j+1\right)} h^{2^{m}}, \\
& (x h)^{2^{m}}=\left(c^{-k} h^{2}\right)^{2^{m-1}}=c^{-k\left(j^{2^{m}-2}+j^{2^{m}-4}+\cdots+j^{2}+1\right)} h^{2^{m}} .
\end{aligned}
$$


There are two conjugacy classes of non-trivial subgroups of $K$ that do not contain $C_{2}^{z}$, represented by any $\left\langle c^{\text {even }} x\right\rangle$ and any $\left\langle c^{\text {odd }} x\right\rangle$, respectively.

Case 1: $\boldsymbol{k}$ even. Then equation (6.3) implies that $c^{-k / 2} h$ has order $2^{m}$ and so without loss of generality, $h$ may be replaced by $c^{-k / 2} h$, which acts trivially on $x$ by conjugation. Thus assume without loss of generality that $k=0$. Also equation (6.4) implies that, in this case, $x h$ has order $2^{m}$, so $h$ can always be replaced by $x h$, which shows that for any $j$, the choices $j$ and $-j$ yield isomorphic groups.

The elements of $\mathscr{H}_{m}$ are isomorphic to $C_{2} \times C_{2^{m}}$. More precisely, a general element of $\mathscr{H}_{m}$ is of the form $\left\langle c^{\delta} x, c^{\gamma} h\right\rangle$ with the two generators commuting and with $c^{\gamma} h$ having order $2^{m}$.

We first claim that if $j= \pm 1$, i.e. if $P=K \times A$, then $G$ has no primitive relations. Indeed, suppose without loss of generality that $j=1$. First note that

$$
\begin{aligned}
\left\langle c^{\delta} x, c^{\gamma} h\right\rangle \in \mathcal{H}_{m} & \Longleftrightarrow\left(c^{\delta} x\right)\left(c^{\gamma} h\right)=\left(c^{\gamma} h\right)\left(c^{\delta} x\right) \\
& \Longleftrightarrow c^{\delta-\gamma} x h=c^{\delta+\gamma} x h \\
& \Longleftrightarrow \gamma \in\left\{0,2^{n-1}\right\} .
\end{aligned}
$$

Let $H_{1}=\left\langle c^{\delta_{1}} x, c^{\gamma_{1}} h\right\rangle, H_{2}=\left\langle c^{\delta_{2}} x, c^{\gamma_{2}} h\right\rangle \in \mathscr{H}_{m}$. If $\delta_{1}=\delta_{2}$ (or indeed if they have the same parity), then $H_{1}$ and $H_{2}$ together generate a proper subgroup of $P$ and so lie in one connected component of $\Gamma$ (see Theorem 2.8, condition (1)). If $\delta_{1} \neq \delta_{2}$ but $\gamma_{1}=\gamma_{2}$, then $H_{1} \cap H_{2}$ has index 2 in each of them and the group $H_{1} H_{2} / H_{1} \cap H_{2}$ is dihedral, so again they lie in the same connected component (Theorem 2.8, condition (2)). Finally, if we have $\delta_{1} \neq \delta_{2}$ and $\gamma_{1} \neq \gamma_{2}$, then $H_{3}=\left\langle c^{\delta_{2}} x, c^{\gamma_{1}} h\right\rangle \in \mathscr{H}_{m}$ also, and the same argument can be applied to the pairs $H_{1}, H_{3}$ and $H_{2}, H_{3}$ to show that $H_{1}$ and $H_{2}$ lie in the same component of $\Gamma$.

Now suppose that $j \neq \pm 1$. We will show that in this case $\operatorname{Prim}(G)=C_{2}$. Replacing $h$ by $x h$ if necessary, we may assume that $j \equiv 3(\bmod 4)$. First, we claim that if $\left\langle c^{\delta} x, c^{\gamma} h\right\rangle \in \mathscr{H}_{m}$, then $\delta \equiv \gamma(\bmod 2)$. Indeed,

$$
\begin{aligned}
\left\langle c^{\delta} x, c^{\gamma} h\right\rangle \in \mathscr{H}_{m} & \Longleftrightarrow c^{\gamma} h c^{\delta} x h^{-1} c^{-\gamma}=c^{2 \gamma+\delta j} x=c^{\delta} x \\
& \Longleftrightarrow 2 \gamma+\delta(j-1) \equiv 0 \quad\left(\bmod 2^{n}\right),
\end{aligned}
$$

which forces $\gamma$ and $\delta$ to have the same parity, since $j-1 \equiv 2(\bmod 4)$. Moreover, for any given $\gamma$, this equation has a solution for $\delta$ and vice versa.

If $H_{1}=\left\langle c^{\delta_{1}} x, c^{\gamma_{1}} h\right\rangle, H_{2}=\left\langle c^{\delta_{2}} x, c^{\gamma_{2}} h\right\rangle \in \mathscr{H}_{m}$ satisfy $\delta_{1} \equiv \delta_{2}(\bmod 2)$, and therefore also $\gamma_{1} \equiv \gamma_{2}(\bmod 2)$, then $H_{1}$ and $H_{2}$ generate a proper subgroup of $P$, so there is an edge between the corresponding vertices of $\Gamma$. It follows that $\Gamma$ has at most two connected components, represented by $H_{\text {odd }}$ with $\delta_{1}$ odd and $H_{\text {even }}$ with $\delta_{2}$ even. It remains to show that two such groups exist in $\mathscr{H}_{m}$ and that 
they lie in distinct connected components of $\Gamma$. Now, there exists an $H_{\text {odd }} \in \mathscr{H}_{m}$ if and only if $c^{\text {odd }} h$ has order $2^{m}$, or equivalently (by (6.3)) $2^{n} \mid\left(j^{2^{m}}-1\right) /(j-1)$, or again equivalently (since $j \equiv 3(\bmod 4)) 2^{n+1} \mid j^{2^{m}}-1$, or equivalently (by Lemma 3.1) $2^{n+1} \mid\left(j^{2}-1\right) 2^{m-1}$. This holds by assumption.

Clearly, $H_{\text {odd }}$ and $H_{\text {even }}$ together generate $P$, so it is enough to show that $H_{\text {odd }} \cap H_{\text {even }}$ is of index greater than 2 in either subgroup. If the intersection was of index 2 , then it would contain an element of order $2^{m-1}$. But $H_{\text {odd }}$ has only two elements of order $2^{m-1}$, namely

$$
e_{1}=\left(c^{\gamma_{1}} h\right)^{2}=c^{\gamma_{1}(j+1)} h^{2} \text { and } e_{2}=c^{\delta_{1}} x\left(c^{\gamma_{1}} h\right)^{2}=c^{\delta_{1}-\gamma_{1}(j+1)} x h^{2},
$$

and similarly for $H_{\text {even }}$. The former is in $H_{\text {even }}$ if and only if

$$
c^{\gamma_{1}(j+1)} h^{2}=c^{\gamma_{2}(j+1)} h^{2} \Longleftrightarrow 2^{n} \mid\left(\gamma_{1}-\gamma_{2}\right)(j+1),
$$

which is impossible since $\gamma_{1}, \gamma_{2}$ have distinct parities and $j$ is assumed to be not equal to -1 in $\mathbb{Z} / 2^{n} \mathbb{Z}$. Similarly, $e_{2}$ is in $H_{\text {even }}$ if and only if

$$
c^{\delta_{1}-\gamma_{1}(j+1)} x h^{2}=c^{\delta_{2}-\gamma_{2}(j+1)} x h^{2} \Longleftrightarrow 2^{n} \mid \delta_{1}-\delta_{2}+\left(\gamma_{2}-\gamma_{1}\right)(j+1),
$$

which is also impossible since $\delta_{1}-\delta_{2}$ is odd, while $\left(\gamma_{2}-\gamma_{1}\right)(j+1)$ is even. This finishes the proof of case (4) of Theorem 1.1.

Case 2: $\boldsymbol{k}$ is odd. In this case no complement of $C \times K$ in $P$ fixes a non-central involution of $K$.

Notation 6.1. Elements of $\mathscr{H}_{m}$ are either isomorphic to $C_{2^{m}}$ and are complements of $C \times K$ in $P$, or isomorphic to $C_{2} \times C_{2^{m-1}}$ and generated by the unique index 2 subgroup of a complement of $C \times K$ in $P$ and by a non-central involution of $K$. We call the two kinds of elements of $\mathscr{H}_{m}$ full image subgroups and half image subgroups, respectively, according to their image in Aut $C$.

We begin by counting the number of elements of $\mathscr{H}_{m}$ of each of the two kinds. An arbitrary full image subgroup of $P$ is of the form $H_{g}=\langle g h\rangle$ for $g \in K$, and conversely, such a group is in $\mathscr{H}$ if and only if $g h$ has order $2^{m}$. By our assumptions (cf. (6.3)), this is satisfied for $g=c^{i}$ for any $i \in \mathbb{Z} / 2^{n} \mathbb{Z}$. Also, by (6.4), $x h$ has order $2^{m}$ if and only if

$$
2^{n} \mid j^{2^{m}-2}+j^{2^{m}-4}+\cdots+j^{2}+1,
$$

which we assume to hold for $j=1$, while for $j \neq 1$ the condition is equivalent to $m>n$ by Lemma 3.1. Since replacing $h$ by $c^{i} h$ for any $i$ changes neither $j$ nor the parity of $k$, the same condition holds for $\left\langle x^{\prime} h\right\rangle \in \mathscr{H}$ for any non-central involution $x^{\prime}$ of $K$. It follows that there are $2^{n}$ full image subgroups of $P$ if $m \leq n$, and $2^{n+1}$ otherwise. 
Next we determine which of these subgroups are conjugate in $P$. The orbit of any full image subgroup $H$ under $P$-conjugation has size $\left|P / N_{P} H\right|=\left|P / C_{P} H\right|$. The last equality follows from the fact that $H$ is a complement in a semi-direct product. Since $H$ does not commute with any non-central involution of $K$, we have

$$
C_{P} H=\left\{c^{a} h^{b} \mid b \in \mathbb{Z} / 2^{m} \mathbb{Z}, a \in \mathbb{Z} / 2^{n} \mathbb{Z}, a(j-1)=0 \in \mathbb{Z} / 2^{n} \mathbb{Z}\right\} .
$$

The centraliser has size $2^{m}\left(j-1,2^{n}\right)$, so the orbit has size $2^{n+1} /\left(j-1,2^{n}\right)$. Note that if $m \leq n$, then our assumptions imply that $j \equiv 3(\bmod 4)$. Indeed, otherwise

$$
v_{2}\left(\left(j^{2^{m-1}}-1\right) /(j-1)\right)=m-1<n,
$$

contradicting the assumption that $2^{n} \mid\left(j^{2^{m-1}}-1\right) /(j-1)$. Thus, if $m \leq n$, there is one orbit of full image subgroups of $P$.

If on the other hand $m>n$, then $H_{x}$ is also a full image subgroup with centraliser

$$
C_{P} H_{x}=\left\{c^{a}(x h)^{b} \mid b \in \mathbb{Z} / 2^{m} \mathbb{Z}, a \in \mathbb{Z} / 2^{n} \mathbb{Z}, a(j+1)=0 \in \mathbb{Z} / 2^{n} \mathbb{Z}\right\} .
$$

Replacing $h$ by $x h$ if necessary, we may assume that $j \equiv 3(\bmod 4)$. Then the orbit of $H=H_{1}$ has size $2^{n}$ and the other orbits have all size $2^{n+1} /\left(j+1,2^{n}\right)$. We deduce that the total number of orbits is

$$
1+\frac{2^{n}}{2^{n+1} /\left(j+1,2^{n}\right)}=1+\frac{\left(j+1,2^{n}\right)}{2} .
$$

From now on, assume without loss of generality that $j \equiv 3$ (mod 4$)$. To summarise the computations, we have

Proposition 6.2. If $m \leq n$, then $H_{1}$ represents the unique conjugacy class of full image subgroups in $\mathscr{H}_{m}$. Otherwise, the distinct conjugacy classes of full image elements of $\mathscr{H}_{m}$ are represented by $\left\{H_{g}\right\}, g \in\left\{1, x, c x, \ldots, c^{\left(j+1,2^{n}\right)-1} x\right\}$.

We now turn to the computation of half image elements of $\mathscr{H}_{m}$. These are of the form

$$
B=\left\langle c^{b} x^{\delta} h^{2}, c^{a} x\right\rangle
$$

Multiplying $c^{b} x^{\delta} h^{2}$ by $c^{a} x$ if necessary we may assume $\delta=0$, and denote the resulting group by $B_{a, b}$. We have

$$
c^{b} h^{2} \cdot c^{a} x \cdot\left(c^{b} h^{2}\right)^{-1} \cdot\left(c^{a} x\right)^{-1}=c^{2 b+k(j+1)+a\left(j^{2}-1\right)} .
$$

The two generators commuting is equivalent to

$$
2 b+k(j+1)+a\left(j^{2}-1\right) \equiv 0 \quad\left(\bmod 2^{n}\right),
$$


which, given any $a \in \mathbb{Z} / 2^{n} \mathbb{Z}$, has two solutions for $b$. This yields at most $2^{n+1}$ half image subgroups. Moreover, since any solution of (6.5) automatically satisfies $v_{2}(2 b) \geq v_{2}(j+1)$, we claim that $c^{b} h^{2}$ has order $2^{m-1}$ (the minimal possible), so that any such $B_{a, b}$ really does represent an element of $\mathscr{H}_{m}$. Indeed,

$$
\left(c^{b} h^{2}\right)^{2^{m-1}}=c^{b\left(j^{2^{m}-2}+j^{2^{m}-4}+\cdots+j^{2}+1\right)}= \begin{cases}c^{\frac{j^{2^{m}}-1}{j^{2}-1}}, & j^{2} \neq 1, \\ c^{b 2^{m-1},}, & j^{2}=1 .\end{cases}
$$

When $j^{2} \not \equiv 1\left(\bmod 2^{n}\right)$,

$$
b \frac{j^{2^{m}}-1}{j^{2}-1}=\frac{j^{2^{m-1}}-1}{j-1} \cdot \frac{b}{j+1} \cdot\left(j^{2^{m-1}}+1\right) .
$$

The three terms on the right have 2 -adic valuations $\geq v_{2}\left(2^{n}\right), \geq-1$ and $\geq 1$ respectively, so the product is a multiple of $2^{n}$. When $j^{2} \equiv 1\left(\bmod 2^{n}\right)$, the same computation together with Lemma 3.1 proves that $b 2^{m-1}$ is a multiple of $2^{n}$, as required. Thus, the half image subgroups of $P$ are precisely $B_{a, b}$ for any solution $a, b$ of (6.5).

Lemma 6.3. There are two conjugacy classes of half image maximal groups in $\mathcal{H}$ : for any $a$ the representatives are $B_{a, b}, B_{a, b^{\prime}}$, where $b, b^{\prime}$ are the two different solutions of (6.5).

Proof. Because conjugation by $h$ acts on the non-central involutions of $K$ as $x \mapsto c^{i} x, i$ odd, and conjugation by $c$ maps $x \mapsto c^{2} x$, all non-central involutions of $K$ are $P$-conjugate. Because the orbit of $c^{a} x$ has length $2^{n}$, its centraliser has size $2^{m+1}$, so it must be $C_{2}^{z} \times B_{a, b}$. Therefore the normaliser of $B_{a, b}$ is of the same size (a normalising element must fix $B_{a, b} \cap K=\left\{1, c^{a} x\right\}$ ), so its orbit has size $2^{n}$ as well.

Finally, we determine which vertices of $\Gamma$ corresponding to the elements of $\mathscr{H}_{m}$ are connected by an edge, and hence the structure of $\operatorname{Prim}(G)$.

Lemma 6.4. If $j=-1$, then the unique full image subgroup of $P$ that intersects a half image subgroup in an index 2 subgroup is $H$. If $j \neq-1$, then there are no such full image subgroups.

Proof. We always have the full image group $H=\langle h\rangle$. The unique index 2 subgroup of $H$ is a subgroup of a half image group if and only if $b=0$ is a solution of (6.5) (for some $a$ ), which it is if and only if $j \equiv-1\left(\bmod 2^{n}\right)$.

Other full image groups (in the case $m>n$ ) are generated by $c^{d} x h$ for some $d$. The square of this element is $c^{d-k-d j} h^{2}$. The exponent $b$ is odd, so it cannot solve (6.5), since $j+1 \equiv 0(\bmod 4)$. 
Proposition 6.5. The group $\operatorname{Prim}(G)$ is isomorphic to

- $\{1\}$ if $m \leq n$ and $j=-1$,

- $C_{2}$ if $m>n$ or $j \neq-1$ but not both,

- $C_{2} \times C_{2}$ if $m>n$ and $j \neq-1$.

Proof. First, note that two half image groups always generate together at most the preimage of the unique index 2 subgroup of Aut $C$, so they always lie in the same connected component of the graph $\Gamma$. A full image group and a half image group together always generate all of $P$ (the group they generate contains a non-central involution of $K$ and a complement to $K$ in $P$, hence also $c$, since $k$ is odd). If $j=-1$, then $H$ intersects a half image subgroup in an index 2 subgroup and the quotient of $P$ by that intersection is dihedral, so in this case, $H$ lies in the same connected component of $\Gamma$ as the half image groups. Proposition 6.2 and Lemma 6.4 now imply that if $m \leq n$, then $\operatorname{Prim}(G)$ is trivial if $j=-1$ and has order 2 otherwise. Suppose that $m>n$. Two full image groups of the form $H_{c^{i} x}$ generate a proper subgroup of $P$, since the subgroup they generate contains no non-central involutions of $K$. On the other hand, $H$ together with any non-conjugate full image subgroup generates $P$ by the same argument as above, and the intersection of two such groups has index greater than 2 , using the fact that $k$ is odd. The claim of the proposition follows.

This finishes the proof of case (5) of Theorem 1.1.

\section{Bibliography}

[1] A. Bartel and T. Dokchitser, Brauer relations in finite groups, J. Eur. Math. Soc. (JEMS), to appear.

[2] S. Bouc, The Dade group of a p-group, Invent. Math. 164 (2006), no. 1, 189-231.

[3] W. Gorenstein, Finite Groups, Chelsea, New York, 1968.

Received December 1, 2011; revised November 25, 2012.

\section{Author information}

Alex Bartel, Mathematics Institute, Zeeman Building, University of Warwick,

Coventry CV4 7AL, United Kingdom.

E-mail: a.bartel@warwick.ac.uk

Tim Dokchitser, Department of Mathematics, University Walk,

Bristol BS8 1TW, United Kingdom.

E-mail: tim.dokchitser@bristol.ac.uk 